\title{
How to Experimentally Monitor the Fatigue Behaviour of Vibrating Mechanical Systems?
}

\author{
Filippo Cianetti* \\ University of Perugia, Italy
}

\begin{abstract}
Fatigue damage and, in general, fatigue behaviour is not simple to observe or estimate during the operational life of a generic vibrating mechanical system. There are a lot of theoretical or numerical methods that allow to evaluate it or by knowing a priori the loading conditions and obtaining output stress states by adopting numerical models of the mechanical system or by directly experimentally measuring and acquiring stress/strain states. A few examples of instruments (e.g. rain flow recorders) or measurement chains dedicated to estimate it in time domain or frequency domain are found in the literature but none that fully both observes the system dynamic behaviour and estimates the related actualized cumulated damage, and, thus, none that can estimate the residual life of the system itself.

In this paper, a simple time-domain method, designed to monitor the instantaneous fatigue behaviour by definition of the instantaneous and cumulated potential damage or of equivalent damage signal amplitude is presented, based on rain-flow counting method and a damage linear cumulation law and starting from system dynamics signals. This methodology was designed to overestimate real damage to alert the system manager before any crack starts and to be simply translated into electronic boards that can be mounted on generic mechanical systems and linked to one of the sensors that usually monitor system functionality.

Keywords: fatigue; damage; rain flow counting; random loads

Highlights

- A smart procedure to on-line evaluate the fatigue behaviour of mechanical systems is presented.

- It is based on rain-flow counting method and a damage linear cumulation law.

- It is designed to be simply translated into electronic boards.

- $\quad$ This method allows placing fatigue among the phenomena to be controlled in feedback in any mechanical system.
\end{abstract}

\section{INTRODUCTION}

Fatigue damage and, in general, fatigue behaviour is not simple to be observed or estimated during the operational life of a generic vibrating mechanical system (e.g. automotive [1] and [2], aeronautical [3] and naval applications [4] or wind turbines [5]). Many theoretical or numerical methods allow evaluating it by knowing a priori the loading conditions (i.e. force or acceleration) [6], expressed in time [6] to [10] or frequency domain [10] to [12], and obtaining output stress states by adopting numerical models of the mechanical system (i.e. multibody (MBS) [13], finite element (FE) [14], multibody with flexible elements (Flex/MBS) [15]) or by directly experimentally measuring and acquiring stress/strain states, knowing, by hypotheses, the fatigue strength (i.e. S-N Wohler or Basquin curve [16]).

A few examples of instruments or measurement chains dedicated to evaluate it in time domain (rain flow recorder [17]) or in frequency domain [18] and [19] are available the in literature but none that fully observes the system dynamic behaviour (i.e. accelerations, internal loads, strains) and foresights the related actualized damage and, is thus able to estimate the residual life of the system itself.
In this paper, a simple time-domain method (used in previous papers [20] and [21]), designed to monitor the instantaneous fatigue behaviour by definition of instantaneous and cumulated potential damage or equivalent damage signal amplitude is presented, based on the Rain Flow Counting (RFC) method [16], on a damage linear cumulation law (PalmegrenMiner's rule [16]), and starting from signals coming from system dynamics. This methodology was designed to overestimate real damage to alert the system manager before any crack starts and to be translated simply into an electronic board to be mounted on a generic mechanical system and linked to one of the sensors that usually monitor system functionality. To this aim, this paper presents its translation in a computing environment dedicated to the dynamic multi-domain simulation of mechanical systems and to the design and verification of control systems. This passage made it possible to verify how the results, obtainable from the evaluation tool, can be obtained online both directly, by the physical measurements made on the turbine, and, in any case, by numerical measures, obtainable through more or less complex dynamic models of the generator itself.

The fundamental hypothesis of this work is that many experimental measures are acquired online, 
for various reasons, on generic machines (i.e. speed, accelerations, moments) and whose values are instantly used to control any condition without being affected by assessments related to the duration, fatigue or damage of the system itself.

Assuming the linear behaviour of the machine and of the mechanical system, a relationship can always be established between these measures and the generic stress state in any location of the components, allowing to drive evaluations toward fatigue on such generic signals but adopting the classical evaluation tools adopted on stress state time histories. If the evaluation of the fatigue behaviour is made starting from a generic signal on which all the hypotheses and tools developed over the years for the evaluation of the damage starting from stress state cannot be directly adopted [16], the definition of fatigue Potential Damage and, therefore, the proposed method can be justified.

\section{TIME DOMAIN FATIGUE ANALYSIS}

If a generic signal (i.e. acceleration, force, moment) $x$ is considered, to evaluate the mechanical system or component fatigue behaviour and to evaluate its durability performance, the fatigue strength curve related to it has to be known. Its expression is the following, similar to that of the Basquin [16] curve for stress signals:

$$
x_{f}=\alpha \cdot N^{\beta},
$$

where $x_{f}$ is the strength amplitude value of the signal related to an applied cycles number $N, \alpha$ is the intercept of the curve on the amplitudes axis for $N=1$, $\beta$ is the curve slope considered constant in the whole cycles range.

Its inverse representation is also valid:

$$
N=\sqrt[\beta]{\frac{x_{a}}{\alpha}},
$$

where $N$ represents the strength cycles number when an amplitude value $x_{a}$ of the alternating signal is applied.

The choice to adopt a single slope curve is justified by the nature of the proposed method intended to monitor potential damage but not real damage. The most common evaluation method of the fatigue behaviour, i.e., of the damage, therefore, requires two further steps: to identify a damage model and to choose a counting and identifying method for the alternating cycles of the signal under examination.
The adoptable damage model is the linear damage cumulation law of Palmegren-Miner [16]. Regarding the cycles counting, the counting method considered as standard in this paper; however, the scientific community and international standards uses the RFC as standard [16]. The RFC identifies the closed hysteretic cycles defined by the signal and, generally, the cycles are collected in bands (bins) to reduce the result dimensions of this evaluation. A load spectrum (i.e. a three-column matrix) can be obtained in which the number of counted cycles $n$, the associated mean value $x_{m}$ and amplitude value $x_{a}$ of the signal are represented in its generic row. All the counted cycles can also still be kept in memory, with relative amplitude and mean value, without to be sampled in bands, obtaining, in this case, a spectrum with as many rows as many cycles were counted, that is assuming for each row $n=1$.

The presence of a mean value would require a further step to adopt the previously mentioned damage model. By adopting, for example, the correction of Goodman or Gerber [16], it is possible to trace back to an equivalent amplitude value of the cycle by knowing ultimate static strength related to of the variable, $x_{u t}$.

However, following the hypothesis introduced in the introduction and at the beginning of this sections, that the signal that is going to be analysed does not allow going back to parameters strictly related to the component strength, for example to the ultimate static strength, the first simplification hypothesis assumed is that the mean value of the generic cycle will be neglected.

Assuming the above hypothesis, the load spectrum can be represented as shown below:

$$
\left(\mathbf{x}_{a}, \mathbf{n}\right),
$$

with, respectively, $\mathbf{x}_{a}$ and $\mathbf{n}$ the vectors of amplitude and number of applied or counted cycles.

By knowing spectrum (Eq. (3)), fatigue damage is evaluable by Palmegren-Miner rule, that is by the following:

$$
D_{p}=\sum_{i=1}^{m}\left[\frac{n_{i}}{\sqrt[\beta]{\frac{x_{a i}}{\alpha}}}\right],
$$

where $m$ is the total number of counted cycles, $D_{p}$ the cumulated damage [16]. Subscript $p$ is used to remember that the damage, not being calculated necessarily starting from a stress value, is potential damage [22] and [23], very useful for comparative 
analysis but not to be analysed as the absolute value of the real damage

Another definition, useful to better understand the subsequent steps proposed by the method object of the present paper, is that of damage equivalent signal (DES) [5], often used in the field of wind engineering.

Under the hypothesis of the constant slope of the fatigue strength curve, by knowing the damage or equivalently the load spectrum, it is possible to define a stationary cyclic condition equivalent to the entire spectrum [23] in terms of damage. Given an arbitrary number of cycles, to which it is possible to assign the value of the total number of cycles $m$, it is always possible to evaluate the equivalent amplitude value $x_{a_{d e s}}$ of the signal that determines the same damage of the whole spectrum $\left(\mathbf{x}_{a}, \mathbf{n}\right)$ by means of the following equation:

$$
x_{a_{d e s}}=\alpha \cdot\left\{m \cdot \sum_{i=1}^{m}\left[\sqrt[\beta]{\frac{x_{a_{i}}}{\alpha}} / n_{i}\right]\right\}^{\beta},
$$

that can be also expressed as follows by adopting damage definition (Eq. (4)):

$$
x_{a_{d e s}}=\alpha \cdot\left[\frac{m}{D_{p}}\right]^{\beta} \text {. }
$$

\section{PROPOSED PROCEDURE FOR DAMAGE MONITORING}

To evaluate the cumulative damage at a given moment in the life of the mechanical system requires acquiring the whole history of the signal, considered representative of its behaviour, from the first use of the machine, seamlessly, to the moment of evaluation. Moreover, it is useful to instantaneously know if the dynamic condition is dangerous or critical for system fatigue behaviour.

The evaluation of cumulated damage is difficult to be performed both for reasons of memory space allocation and for reasons related to computational times to perform cycles counting through RFC and then to damage evaluation. As concerns the second aim, an instantaneous damage definition does not exist, such as an instantaneous equivalent damage value of the reference signal to be adopted to control system dynamics actively.

The author wants to demonstrate the possibility of monitoring the potential damage of a generic machine by evaluating it at any of the operating times without taking up all the memory space required by the ideal methodology, evaluating it by adopting a mobile window defined in the time domain, of appropriate characteristics, allowing by this approach to define the "instantaneous" damage such as the "instantaneous" equivalent damage signal.

Let us imagine having a signal measured throughout its temporal extension $T$ and on which it is, therefore, possible to evaluate the real potential damage by applying the RFC and Palmgren-Miner's rule (Eq. (4)) in increasing time intervals $\left[0, t_{i}\right]$, with $t_{i}$ between 0 and $T$. This enables defining a time history of the damage $D_{p}(t)$. Associated with this time history, the time history of the damage equivalent signal $x_{a_{d e s}}(t)$ can also be obtained. Fig. 1 shows the flow chart of the process.

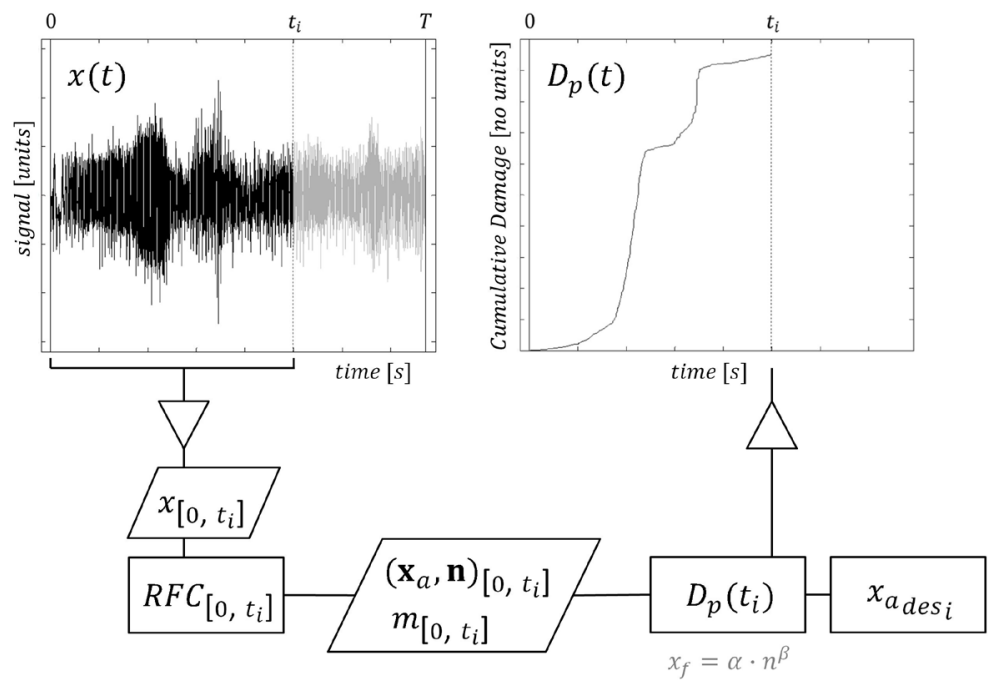

Fig. 1. Flow chart of standard evaluation of damage time history [20] 
The formulation of the RFC method is a function of the hypothesis that the signal time history is single or repeats itself several times, which introduces a dichotomy of the load spectrum and, therefore, of the damage and of the equivalent alternating value of the signal caused by the definition of residue [16] and [24] and its management within the counting method. The residues are the values of the signal that do not determine any closed hysteretic cycle and, therefore, are not considered in the evaluation of the damage for single signal time history. For signals that repeats several times, however, by adopting the rule of Cloorman-Seeger or ASTM [24] and [25], all the cycles are forcibly closed, which determines a different assessment of the damage for the two aforementioned hypotheses. With the objective of monitoring fatigue in mechanical systems that are typically very long-term loaded with variable and random loads, the hypothesis of repeating signal is adopted in this paper and in this proposed approach.

The first step of the proposed procedure is to define a moving window.

Which sampling time $d t$ should has to be taken? How long must the mobile window be $\Delta T$ ?

The answers to these two questions define the fundamental choices of the procedure.

Answering these questions means analysing the mechanical system and the load conditions to which it is typically subjected and, therefore, presumably, to which it will be subjected in the future, during operating conditions.

The analysis of the frequency content of the loads (accelerations or forces) and of the natural frequencies of the system and/or component constitutes the instrument with which to reach and define these two values.

The sampling time must be such as to capture the maximum frequency $f_{\max }$ that is wanted to be observed, whether this is the maximum observable in the input or that represents the natural mode of maximum natural frequency that is to be considered. The sampling time $d t$ must be $k$ times lower than that corresponding to the maximum frequency value:

$$
d t=\frac{1}{k \cdot f_{\max }} .
$$

The length of the mobile window $\Delta T$ must instead be such as to capture the minimum frequency $f_{\min }$ that is wanted to be observed, whether this is the minimum observable in the input or that represents the natural mode of minimum natural frequency that is to be considered. $\Delta T$ must be at least $k$ times the value of the one corresponding to the minimum frequency:

$$
\Delta T=k \cdot \frac{1}{f_{\min }} .
$$

These two limit values allow observing and, therefore, being able to count the cycles associated with both fast and slow phenomena in the mobile window, in an appropriate number that can represent a significant spectrum for the loading condition.

Once the floating window has been defined, this is the data buffer that is continuously filled in for the evaluation of fatigue behaviour.

In Fig. 2, a flow chart of the proposed procedure is shown.

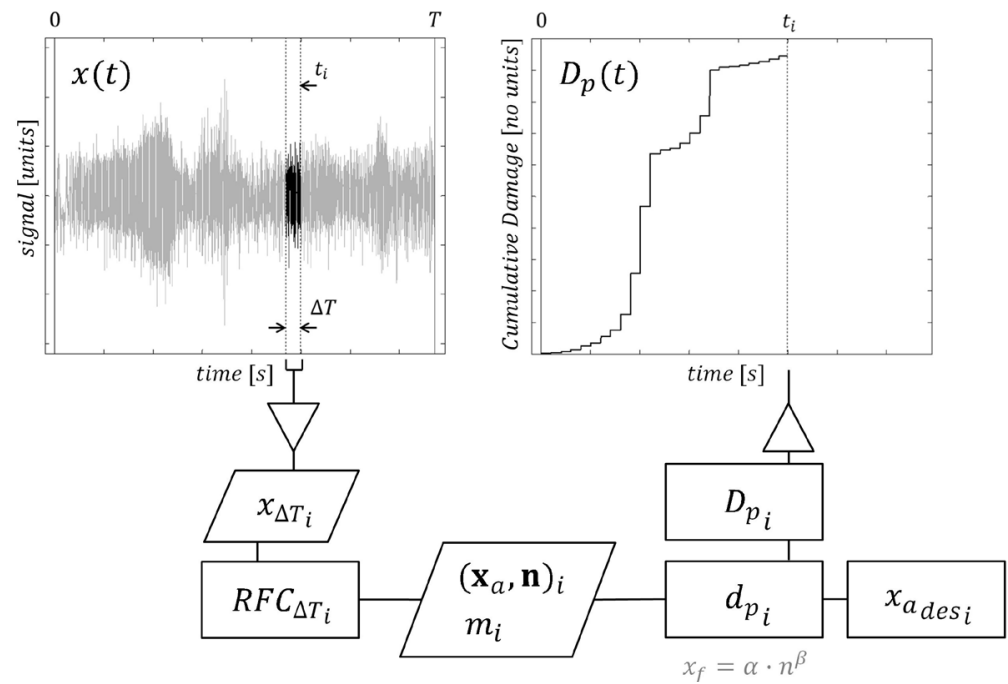

Fig. 2. Flow chart of proposed evaluation of damage time history [20] 
When the mobile $i^{\text {th }}$ window is post-processed, the load spectrum obtained by RFC is:

$$
\left(\mathbf{x}_{a}, \mathbf{n}\right)_{i} .
$$

The Cloorman-Seeger hypothesis is followed without considering the cycle mean value.

If a strength curve such as Eq. (1) is adopted, it is possible to define the $i^{\text {th }}$ potential damage $d_{p}$, which is called instantaneous damage, meaning by instantaneous the one associated with the current mobile window:

$$
d_{p_{i}}=\sum_{k=1}^{m_{i}}\left[\frac{\left(n_{k}\right)_{i}}{\sqrt[\beta]{\frac{\left(x_{a_{k}}\right)_{i}}{\alpha}}}\right],
$$

in which subscript $i$ refers to $i^{\text {th }}$ window and $k$ to the generic spectrum cycle (Eq. (9)), counted in the same window. $m_{i}$ is the total number of cycles counted in the window.

The cumulated damage at the generic instant, that is at the generic $i^{\text {th }}$ window, is:

$$
D_{p_{i}}=\sum_{r=1}^{i} d_{p_{i}}
$$

Similarly, the DES related to the window is:

$$
\begin{aligned}
& x_{a_{d e s i}}=\alpha \cdot\left\{m_{i} \cdot \sum_{k=1}^{m_{i}}\left[\sqrt[\beta]{\frac{\left(x_{a_{k}}\right)_{i}}{\alpha}} /\left(n_{k}\right)_{i}\right]\right\}^{\beta}, \\
& x_{a_{d e s i}}=\alpha \cdot\left[\frac{m_{i}}{d_{p_{i}}}\right]^{\beta} \text {. }
\end{aligned}
$$

The value $x_{a_{d e s}}$ is strongly influenced by the number of cycles counted in the window, $m_{i}$, and therefore window by window, could vary in value, increasing or decreasing, without, however, meaning that the damage has increased or decreased. For example, if two windows $i^{\text {th }}$ and $(i+1)^{\text {th }}$ generate the same instantaneous damage $d_{p}$ but the two windows contain different numbers of cycles $m_{i}$ and $m_{i+1}$, two different values of $x_{a_{d e s}}$ occur for the same damage. To overcome this result and have a value of comparable among the various windows and, therefore, independent of the number of cycles, the value of the normalized DES has been defined $\bar{x}_{a_{d e s}}$ that is evaluated in the hypothesis of a number of cycles constant for all the windows. In the case of the number of cycles constant and equal to 1 its definition is:

$$
\begin{gathered}
\bar{x}_{a_{d e_{i}}}=\alpha \cdot\left\{\sum_{k=1}\left[\sqrt[m_{i}]{\frac{\beta}{\alpha}} /\left(n_{k}\right)_{i}\right]\right\}^{\beta}, \\
\bar{x}_{a_{a_{k s i}}}=\alpha \cdot\left[d_{p_{i}}\right]^{-\beta} .
\end{gathered}
$$

It has to be highlighted that the definition of the fatigue curve as previously done and the consequent damage evaluation procedure are strictly related. If we have to manage signals that are not stresses or strains and thus not directly related to the concept of the Basquin curve or of hot spot stress or of the damage rule, a virtual damage evaluation has to be accepted, which that means the definition of a strength curve that implicitly considers aspects such as stress concentration, mean effect, or reliability. As more these are well modelled into the curve, the potential damage and the instantaneous damage will be closer to the real one.

\section{SIMPLE TEST CASE}

The example (signal) considered to test the goodness of the method is shown in Fig. 3 and relative to an accelerometric measurement carried out in a wind tunnel, on a mini-wind generator [20], [21] and [26]. It varies in a rage from $2 \mathrm{~m} / \mathrm{s}^{2}$ to $-2 \mathrm{~m} / \mathrm{s}^{2}$.

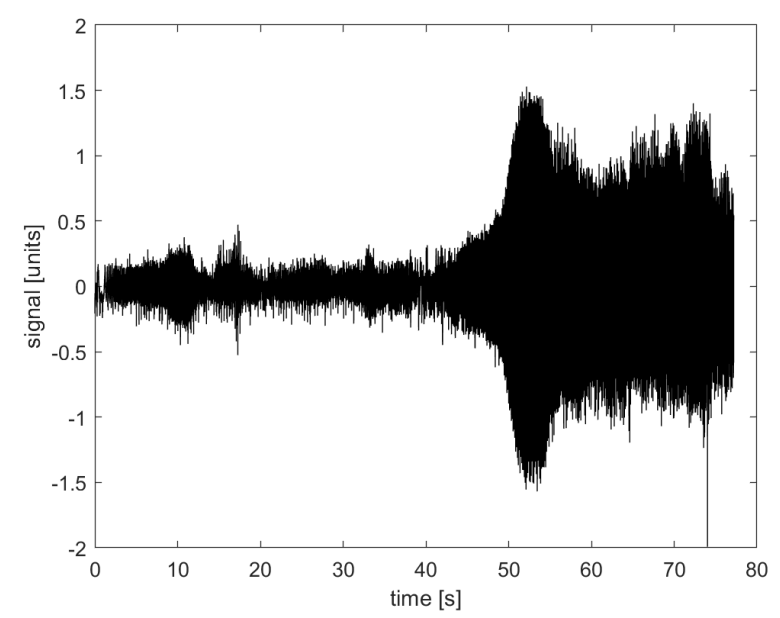

Fig. 3. Time history of signal test case

Figs. 4 and 5 show the rain flow matrix, the cumulative of the amplitudes of the cycles (Fig. 4) and the time histories of the equivalent signal (DES) and of the damage (Fig. 5). The calculation of the damage and of the equivalent signal was carried out by assuming a fatigue strength curve with parameters $\alpha=19.247 \mathrm{~m} / \mathrm{s}^{2}$ and $\beta=-0,2228$. 
a)

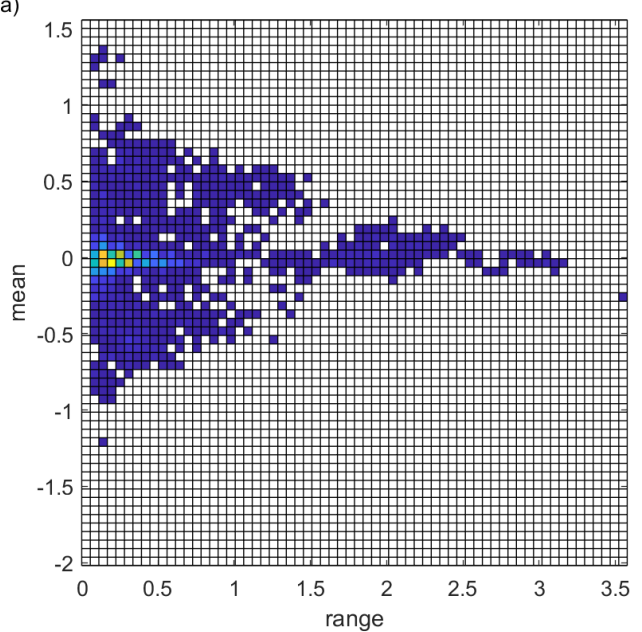

b)

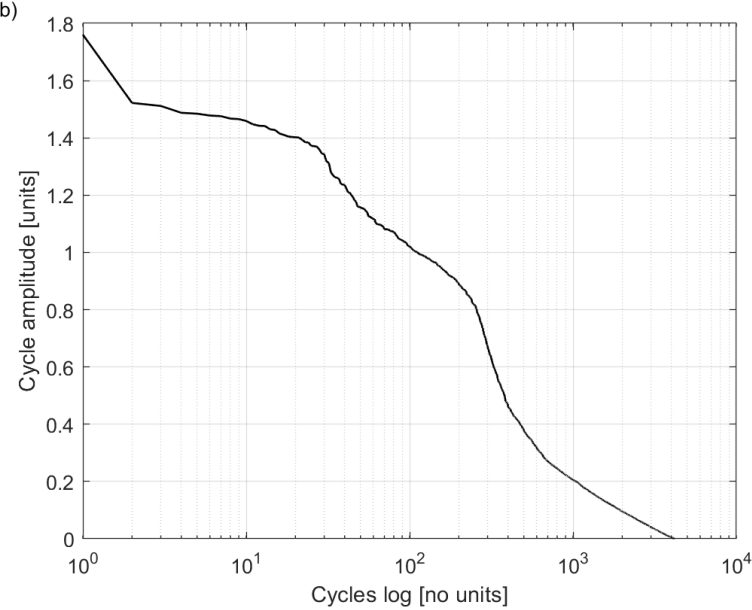

Fig. 4. a) Rain flow counting matrix, and b) cycles amplitude cumulative of test case time history

a)

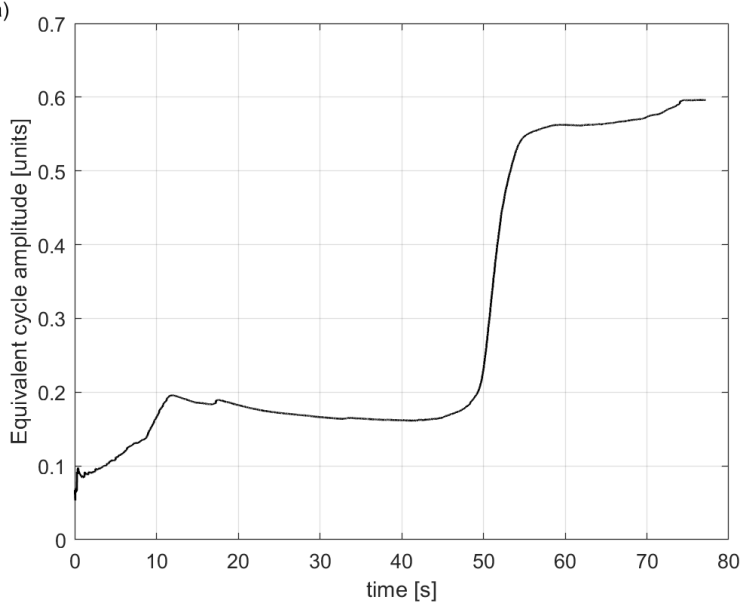

b)

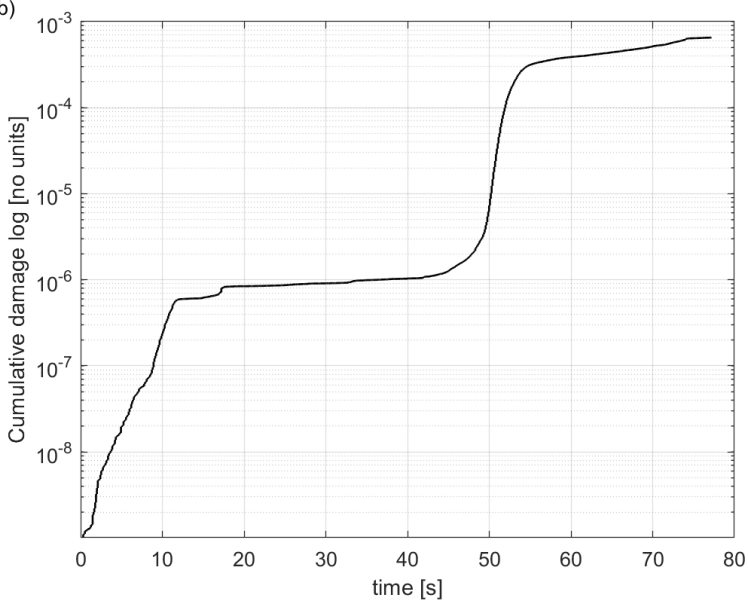

Fig. 5. a) Damage equivalent signal and b) cumulative damage time histories of test case signal

a)

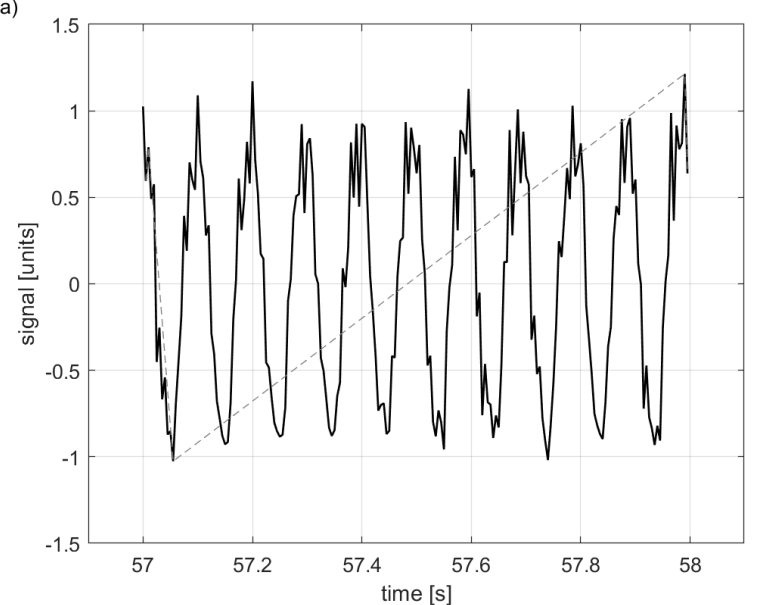

b)

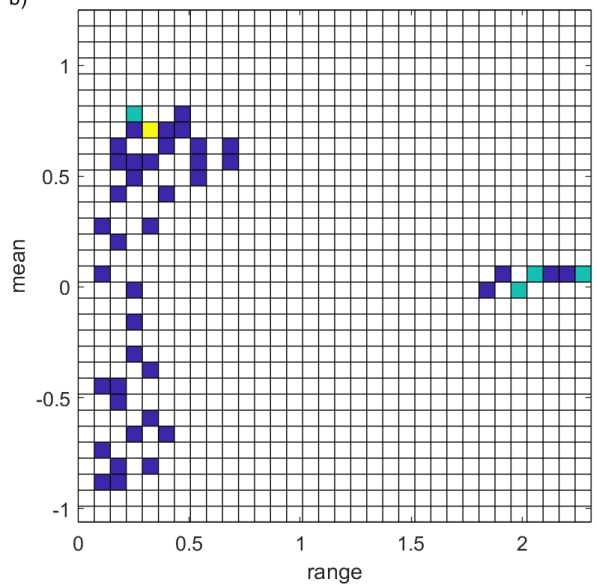

Fig. 6. a) Example of $i^{\text {th }}$ signal time window of proposed process $(i=58)$, and $\left.b\right)$ its rain flow matrix 

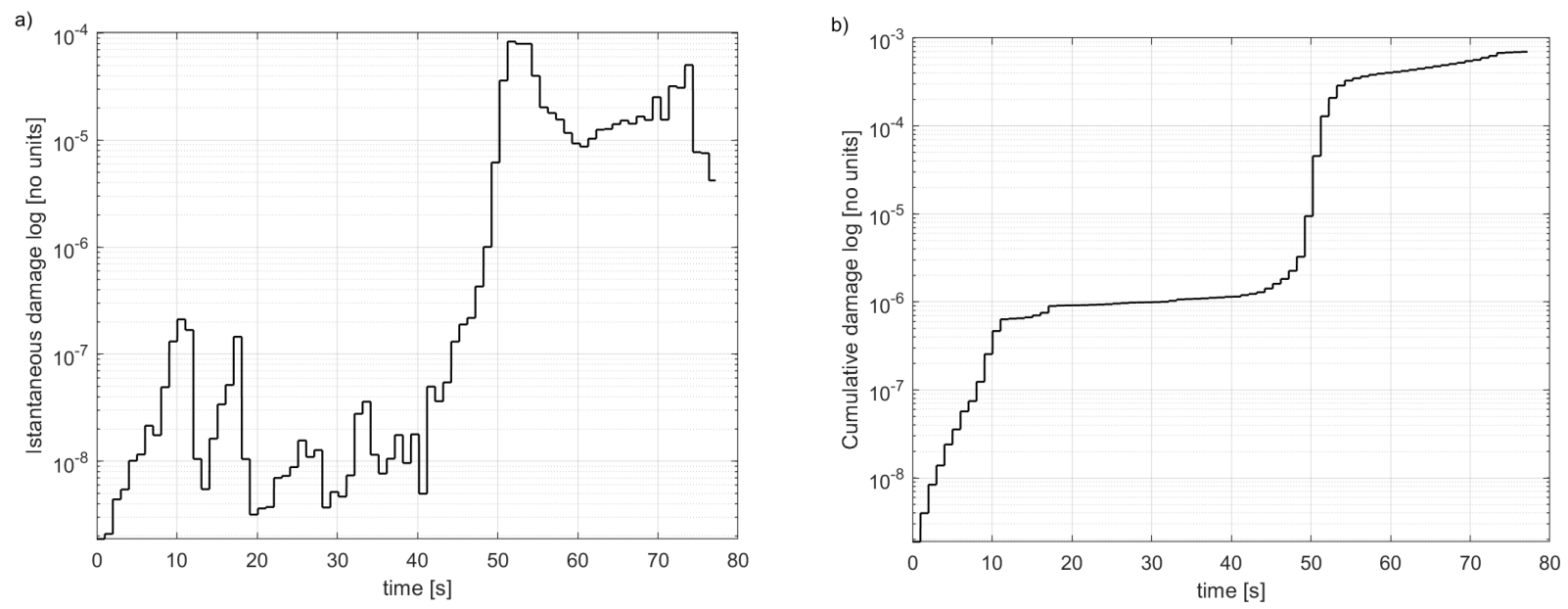

Fig. 7. Proposed method results; a) instantaneous damage $d_{p}$, and b) cumulative damage $D_{p}$ time histories
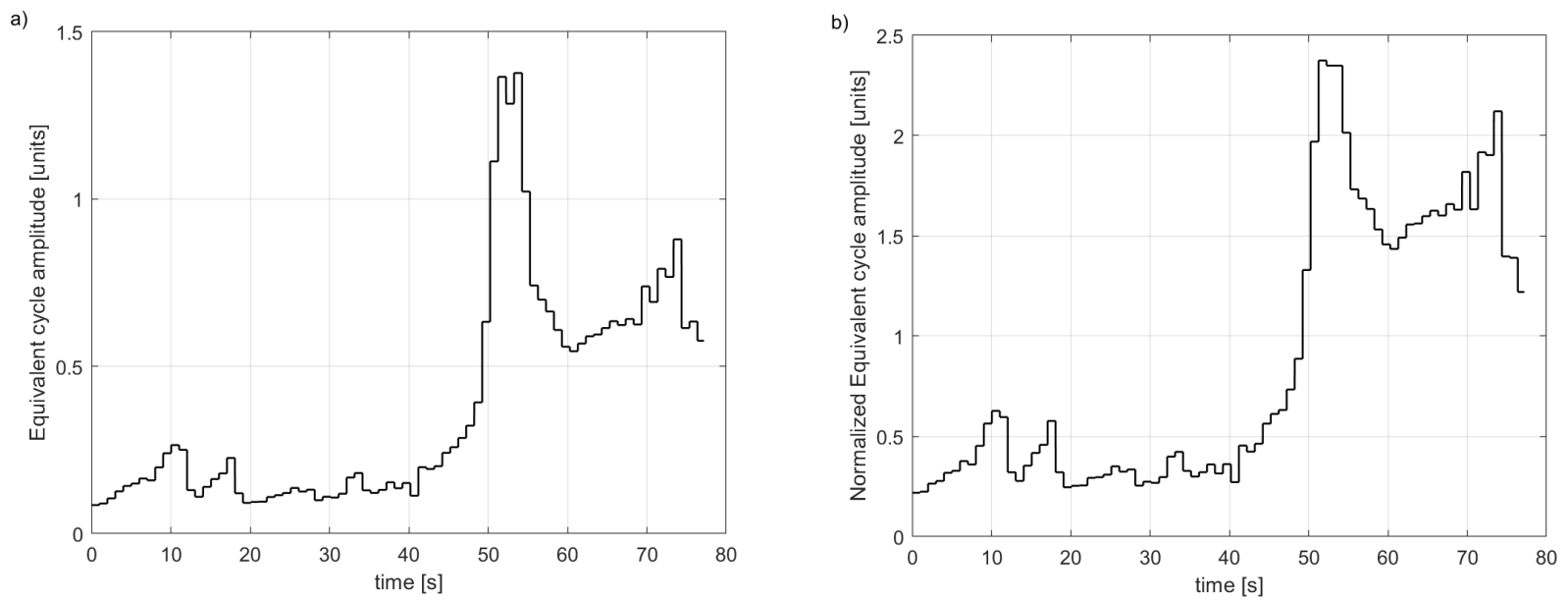

Fig. 8. Proposed method results; a) damage equivalent signal amplitude $x_{a_{d e s}}$, and b) normalized damage equivalent signal amplitude $\bar{x}_{a_{d e s}}$ time histories

Analysing the temporal profile of the wind speed, which in this case was constant (therefore without a defined frequency content), the minimum and maximum frequencies were defined exclusively starting from the natural frequencies of the tower [26]. Considering as a minimum factor a factor $k$ (Eqs. (7) and (8)) equal to 5 , a mobile time window has been defined, characterized by $\Delta T=1.0 \mathrm{~s}$ and $d t=0.005 \mathrm{~s}$, which satisfies both relations (Eqs. (7) and (8)).

Fig. 6 shows one of the moving windows $(i=58)$ corresponding to an absolute time $t_{i}$ (Fig. 2) equal to $58 \mathrm{~s}$ and the relative Rain Flow matrix; Fig 7 shows the instantaneous damage $\left(d_{p}\right)$ and the cumulated one $\left(D_{p}\right)$ evaluated over the whole test time history.

In order to understand the evaluation of DES carried out following the hypothesis adopted in Eqs. (14) and (15), Fig. 8 shows the time histories of DES obtained by considering the real number of cycles per window $m_{i}$ (see Eqs. (12) and (13)) and considering it equal to 1 (see Eqs. (14) and (15)).

In the end, the time history of the real cumulated damage (Fig. 5) was compared with the corresponding trend obtained by means of the proposed methodology (Fig. 9). The trend of the instantaneous damage $d_{p_{i}}$ obtained from the proposed methodology cannot be compared to a similar real value since the damage cannot be defined in absolute terms as an instantaneous value. In order to carry out a qualitative comparison, however, $d_{p_{i}}$ was compared with the first derivative of the real cumulated damage $d D_{p} / d t$ (Fig. 9). A comparison between the equivalent signals (DES) is not possible since, by definition, while for the real signal the number of cycles $m$ grows monotonously over time (see Fig. 1) in the sampled one (proposed 

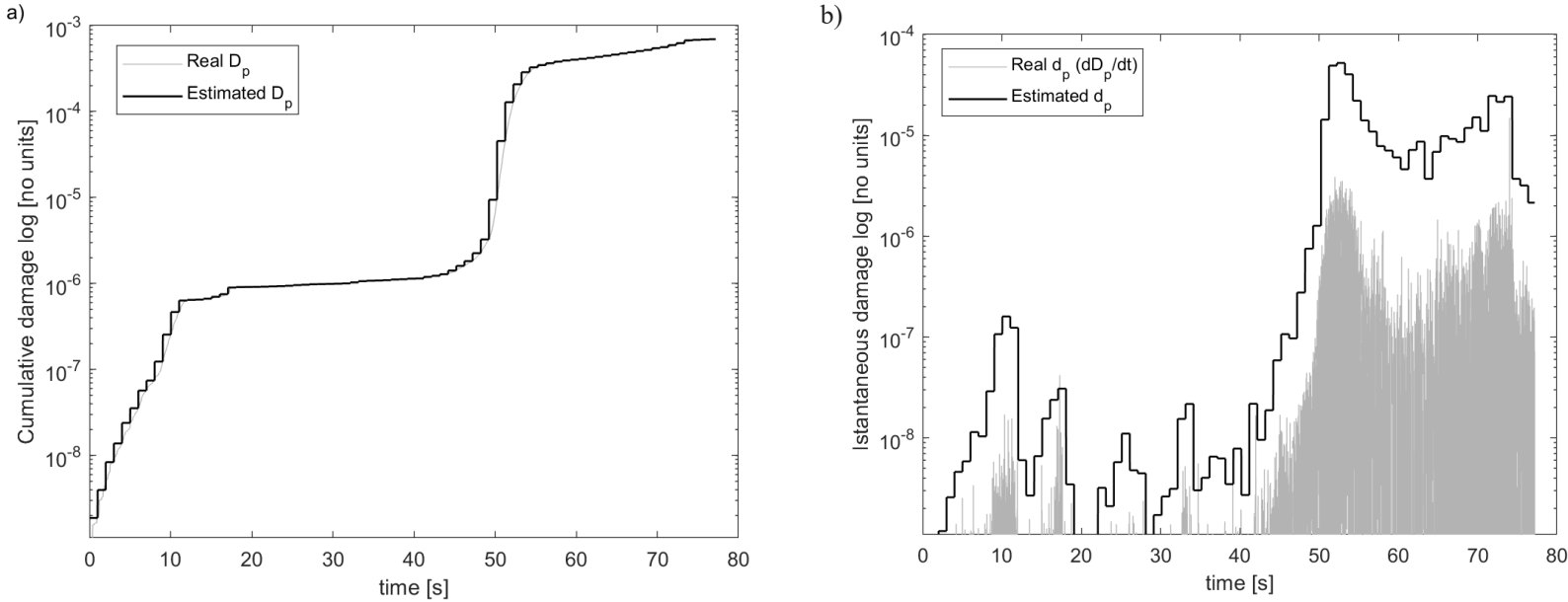

Fig. 9. Comparison between results obtained by proposed method (Estimated) and standard one (Real); a) cumulative damage $D_{p}$, and b) instantaneous damage $d_{p}$ time histories
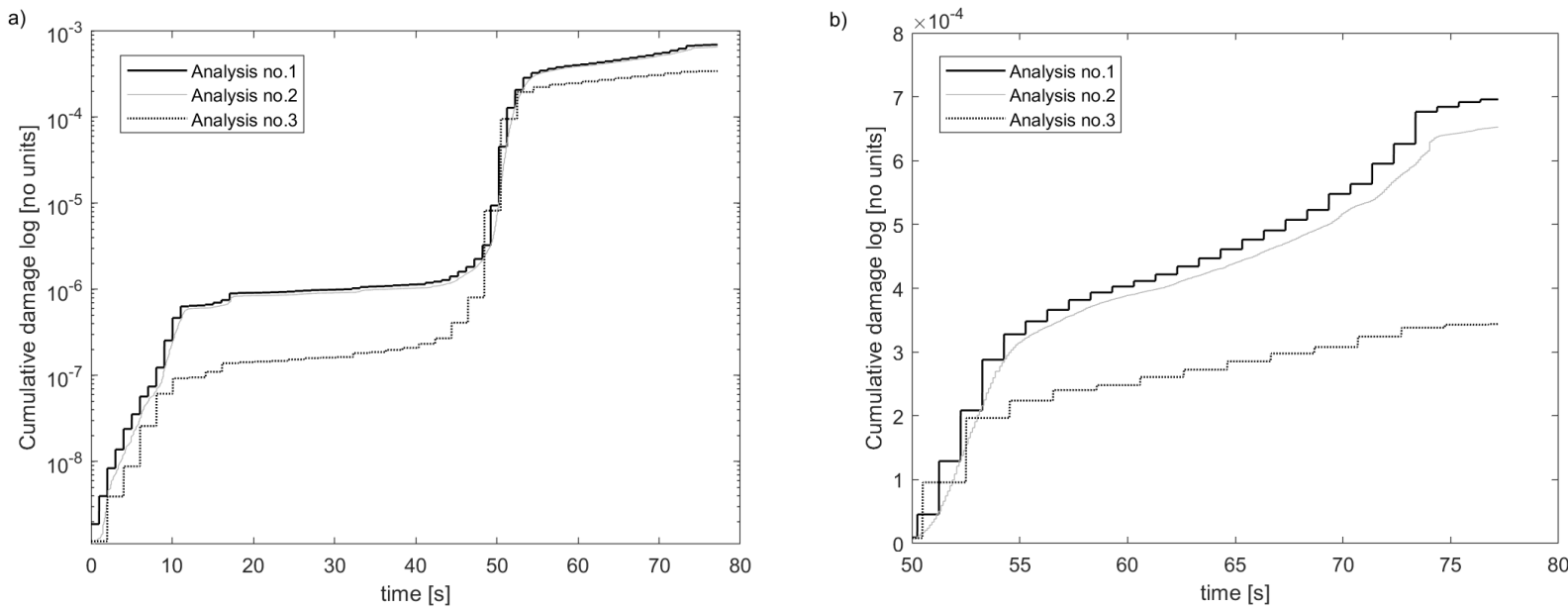

Fig. 10. Proposed method sensitivity analysis; potential damage cumulatives; a) comparison among results obtained by adopting windowing sizes of Table 1, b) detailed comparison (in linear scale) for the last 30 seconds of the signal
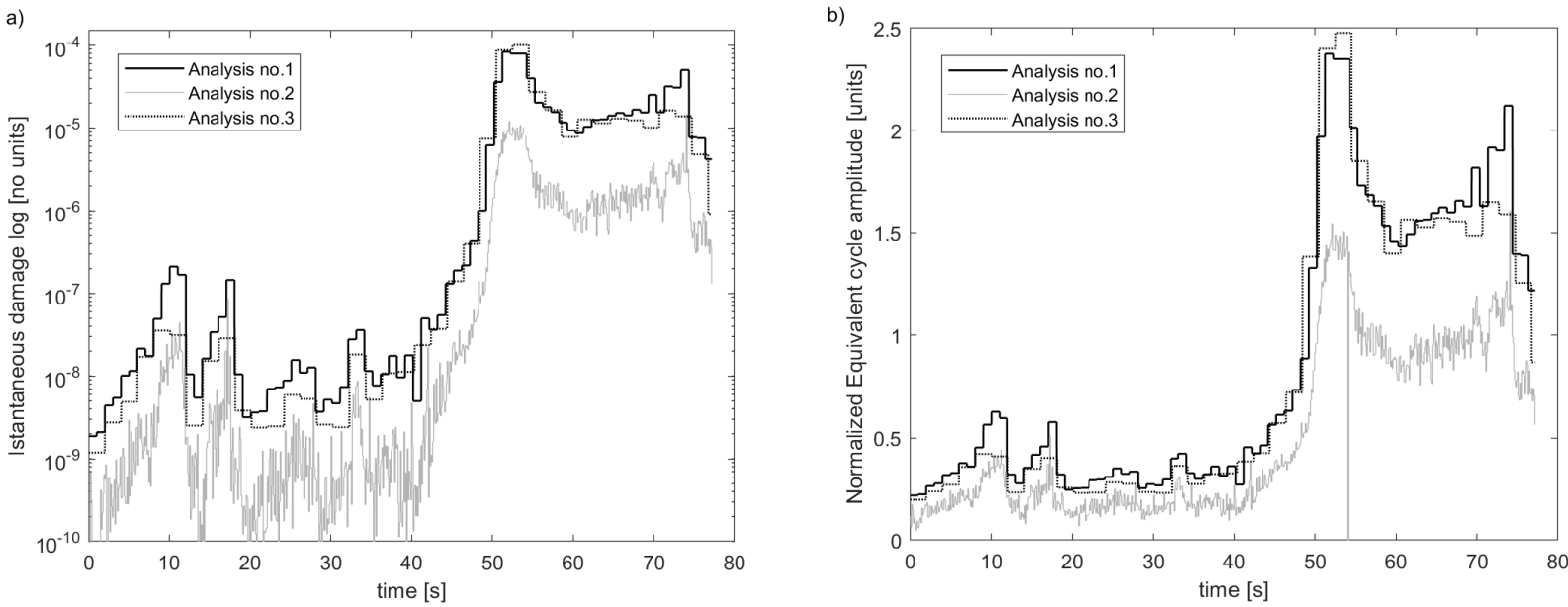

Fig. 11. Proposed method sensitivity analysis; comparison among results obtained by adopting windowing sizes of Table 1; a) instantaneous damage and b) normalized DES 
method) each window shows a number of increasing or decreasing variable cycles (see Fig. 2).

The comparison between the cumulative damage time histories (Fig. 9) shows how the proposed method is sufficiently accurate to estimate both the trend but also the absolute value of the damage.

In the final part of the paper, how the choice of an incorrect sampling time rather than an incorrect size of the mobile window can negatively affect the obtainable results is shown.

In Figs. 10 and 11, the results previously obtained with $\Delta T=1.0 \mathrm{~s}$ and $d t=0.005 \mathrm{~s}$ are compared with those obtained with other two pairs of values of $\Delta T$ and $d t$ for which values too small of $\Delta T$ have been deliberately adopted, from not being able to count the low-frequency cycles accurately (in this test case the most important), and too large values of $d t$ have also adopted, losing the small-amplitude cycles (in this test case, the greater number) (Table 1).

Table 1. Windowing parameters adopted for sensitivity analysis

\begin{tabular}{llc}
\hline & \multicolumn{2}{c}{ Mobile window parameters } \\
\cline { 2 - 3 } & $d t[\mathrm{~s}]$ & $\Delta T[\mathrm{~s}]$ \\
\hline Analysis no.1 & 0.005 & 1.00 \\
\hline Analysis no.2 & 0.005 & 0.10 \\
\hline Analysis no.3 & 0.020 & 1.00 \\
\hline
\end{tabular}

Fig. 10 compares the time histories of the cumulative damage, real and estimated by the proposed method (represented in a logarithmic scale on the left and in a linear scale and for the final part of the signal on the right). In Fig. 11, the trends of the instantaneous damage (Fig. 11a) and of the normalized equivalent signal (Fig. 11b) are instead compared.

It can be noted that the choice of these two parameters significantly influences the results.

\section{CONCLUSIONS}

In this paper, it has been demonstrated how, starting from the online measurement of any representative signal of the behaviour of the mechanical system, it is possible to define and obtain an instantaneous evaluation of the potential damage of the signal in terms of damage (DES) in the time domain. This possibility provides the scientific and technical community the automatic control the possibility to insert the fatigue among the phenomena to be controlled in feedback in any mechanical system (i.e., automotive vehicles, aircraft, trains, ships, wind turbines) evaluating not only the maximum or minimum values in the signal but also their damaging potential.

In this way, in addition to an instantaneous evaluation, a cumulative evaluation of the potential damage is also obtained, which becomes a further control parameter, given an admissible threshold for this signal.

The author does not want to estimate the final damage related to an assigned time duration of the system by observing a single time window (in this case, that has to be sufficiently long to stabilize damage variance) but only to evaluate an "instantaneous" one and obtain the actual cumulated damage by cumulating these values, which is a characteristic of the approach that allows it to be used as a monitor.

The proposed methodology highlights how particular attention must be paid to the choice of the characteristic parameters of the window, specifically, its time length and sampling time. The results obtained in the final part of the paper show how, although in absolute value the result is influenced by this choice, the trends of the cumulative damage, as well as of the instantaneous one and of the DES, are very similar to different window parameters.

\section{ACKNOWLEDGEMENTS}

This research activity was financed by Italian PRIN funding source (Research Projects of National Interest - Progetti di Ricerca di Interesse Nazionale) by a financed project entitled SOFTWIND (Smart Optimized Fault-Tolerant WIND turbines).

\section{REFERENCES}

[1] Ashmore, S.C., Piersol, A.G., Witte, J.J. (1992). Accelerated service life testing of automotive vehicles on a test course. Vehicle System Dynamics, vol. 21, no. 2, p. 89-108, DOI:10.1080/00423119208969004.

[2] Liu, J., Wang, Y., Li, W. (2010). Simplified fatigue durability assessment for rear suspension structure. International Journal of Automotive Technology, vol. 11,. p. 659-664, DOI:10.1007/s12239-010-0078-1.

[3] Gao, Q., Wang, Y., Zhang, X. (2011). Prediction of aeronautic rescue mechanism under random vibration based on sample approach. Zhongguo Jixie Gongcheng/China Mechanical Engineering, vol. 22, vol. 2, p. 162-165.

[4] Wang, Y. (2010). Spectral fatigue analysis of a ship structural detail - A practical case study. International Journal of Fatigue, vol. 32, no. 2, p. 310-317, D0l:10.1016/j. ijfatigue.2009.06.020.

[5] Corradini, M.L., Ippoliti, G., Orlando, G. (2018). Fault-tolerant sensorless control of wind turbines achieving efficiency maximization in the presence of electrical faults. Journal 
of the Franklin Institute, vol. 355, no. 5, p. 2266-2282, DOl:10.1016/j.jfranklin.2018.01.003.

[6] Braccesi, C., Cianetti, F., Tomassini, L. (2016). An innovative modal approach for frequency domain stress recovery and fatigue damage evaluation. International Journal of Fatigue, vol. 91, p. 382-396, D0l:10.1016/j.ijfatigue.2016.02.028.

[7] Braccesi, C., Morettini, G., Cianetti, F., Palmieri, M. (2018). Development of a new simple energy method for life prediction in multiaxial fatigue. International Journal of Fatigue, vol. 112, p. 1-8, D0l:10.1016/j.ijfatigue.2018.03.003.

[8] Carpinteri, A., Spagnoli, A., Vantadori, S. (2017). A review of multiaxial fatigue criteria for random variable amplitude loads. Fatigue \& Fracture of Engineering Materials \& Structures, vol. 40, no. 7, p. 1007-1036, D0l:10.1111/ffe.12619.

[9] Braccesi, C., Cianetti, F., Lori, G., Pioli, D. (2014). Evaluation of mechanical component fatigue behavior under random loads: Indirect frequency domain method. International Journal of Fatigue, vol. 61, p. 141-150, D0l:10.1016/j. ijfatigue.2013.11.017.

[10] Łagoda, T., Macha, E., Niesłony, A. (2004). Comparison of the rain flow algorithm and the spectral method for fatigue life determination under uniaxial and multiaxial random loading. Journal of ASTM International, vol. 1, 8, p. 1-13, D0l:10.1520/ JAI19031.

[11] Braccesi, C., Cianetti, F., Lori, G., Pioli, D. (2015). Random multiaxial fatigue: A comparative analysis among selected frequency and time domain fatigue evaluation methods. International Journal of Fatigue, vol. 74, p. 107-118, D0I:10.1016/j.ijfatigue.2015.01.003.

[12] Benasciutti, D., Sherratt, F., Cristofori, A. (2016). Recent developments in frequency domain multi-axial fatigue analysis. International Journal of Fatigue, vol. 91, p. 397-413, D0I:10.1016/j.ijfatigue.2016.04.012.

[13] Braccesi, C., Cianetti, F., Landi, L. (2005). Random loads fatigue: The use of spectral methods within multibody simulation. Proceedings of the ASME International Design Engineering Technical Conferences and Computers and Information in Engineering Conference, vol. 1, p. 1735-1745, DOI:10.1115/DETC2005-84453.

[14] Preumont, A., Piefort, V. (1994). Predicting random highcycle fatigue life with finite elements. Journal of Vibration and Acoustics, Transactions of the ASME, vol. 116, no. 2, p. 245248, DOI:10.1115/1.2930420.

[15] Braccesi, C., Cianetti, F. (2005). A procedure for the virtual evaluation of the stress state of mechanical systems and components for the automotive industry: Development and experimental validation. Proceedings of the Institution of Mechanical Engineers, Part D: Journal of Automobile Engineering, vol. 219, no. 5, p. 633-643, D0I:10.1243/095440705X11040.

[16] Collins, J.A. (1981). Failure of Materials in Mechanical Design, Wiley, New York.

[17] Murakami, Y., Morita, T., Mineki, K. (1997). Development and application of super-small size strain history recorder based on rainflow method. Journal of the Society of Materials Science Japan, vol. 46, no. 10, p. 1217-1221, D0l:10.2472/ jsms.46.1217. (in Japanese)

[18] Braccesi, C., Cianetti, F., Moretti, M., Rossi, G. (2015). Random loads fatigue. Experimental approach through thermoelasticity. Procedia Engineering, vol. 101, p. 312-321, D0I:10.1016/J.proeng.2015.02.038.

[19] Zanarini, A. (2015). Full field experimental modelling in spectral approaches to fatigue predictions. Proceedings of IFTOMM - ICOEV2015 International Conference on Engineering Vibration, p. 773- 782.

[20] Cetrini, A., Cianetti, F., Corradini, M.L., Ippoliti, G., Orlando, G. (2019). On-line fatigue alleviation for wind turbines by a robust control approach. International Journal of Electrical Power and Energy Systems, vol. 109, p. 384-394, D0I:10.1016/j. ijepes.2019.02.011.

[21] Cianetti, F., Cetrini, A., Corradini, M.L., Ippoliti, G., Orlando, G. (2018). Dynamic behavior of wind turbines. An onboard evaluation technique to monitor fatigue. Procedia Structural Integrity, vol. 12, p. 102-112, D0l:10.1016/j. prostr.2018.11.103.

[22] Cianetti, F., Alvino, A., Bolognini, A., Palmieri, M., Braccesi, C. (2018). The design of durability tests by fatigue damage spectrum approach. Fatigue and Fracture of Engineering Materials and Structures, vol. 41, no. 4, p. 787-796, DOI:10.111//ffe.12686.

[23] Cianetti, F. (2012). Development of a modal approach for the fatigue damage evaluation of mechanical components subjected to random loads. SDHM Structural Durability and Health Monitoring, vol. 8, no. 1, p. 1-29.

[24] Clormann, U., Seeger, T. (1986). Rainflow - HCM - Ein Zählverfahren für Betriebsfestigkeitsnachweise auf werkstoffmechanischer Grundlage. Stahlbau, vol. 55, p. 65117.

[25] ASTM Standard E 1049 (1985). Standard Practices for Cycle Counting in Fatigue Analysis. ASTM International, West Conshohocken.

[26] Castellani, F., Astolfi, D., Becchetti, M., Berno, F., Cianetti, F., Cetrini, A. (2018). Experimental and numerical vibrational analysis of a horizontal-axis micro-wind turbine. Energies, vol. 11, no. 2, art. ID 456, D0l:10.3390/en11020456. 